\title{
A Framework for Group Decision-Making: Including Cognitive and Affective Aspects in a MCDA Method for Alternatives Rejection
}

\author{
João Carneiro ${ }^{1}$, Luís Conceição ${ }^{1}$, Diogo Martinho ${ }^{1}$, Goreti Marreiros ${ }^{1}$ and Paulo \\ Novais $^{2}$ \\ ${ }^{1}$ GECAD - Research Group on Intelligent Engineering and Computing for Advanced \\ Innovation and Development, Institute of Engineering, Polytechnic of Porto, Porto, Portugal \\ \{jomrc, lmdsc, diepm, mgt\}@isep.ipp.pt \\ ${ }^{2}$ ALGORITMI Centre, University of Minho, Braga, Portugal \\ pjon@di.uminho.pt
}

\begin{abstract}
With the evolution of the organizations and technology, Group Decision Support Systems have changed to support decision-makers that cannot be together at the same place and time to make a decision. However, these systems must now be able to support the interaction between decision-makers and provide all the relevant information at the most adequate times. Failing to do so may compromise the success and the acceptance of the system. In this work it is proposed a framework for group decision using a Multiple Criteria Decision Analysis method capable of identify inconsistent assessments done by the decision-maker and identify alternatives that should be rejected by the group of decision-makers. The proposed framework allows to present more relevant information throughout the decision-making process and this way guide decisionmakers in the achievement of more consensual and satisfactory decisions.
\end{abstract}

Keywords: MCDA, Consensus-based Approach, Group Decision Support Systems, Group Decision-Making, Cognitive Decision-Making.

\section{Introduction}

Decision-making has always been a core process of any organization [1,2]. Nowadays, most of the decisions taken inside organizations are made in group [1].There are many advantages associated to group decision-making which allow better decisions to be made [3]. For instance, Dennis [4] stated some advantages, such as: to share workloads, to build social networks, to gain support among stakeholders, to train less experienced group members and most importantly to improve the quality of the decision. Other advantages include more knowledge being exchanged between decision-makers, better evaluation of the alternatives (compared to individual decisionmaking), increased acceptance of a decision and a better comprehension of the problem and the decision [5-7]. Group Decision Support Systems (GDSS) have been studied 
throughout the last decades with the objective of supporting decision-makers in group decision-making processes. With the appearance of global markets, the growth of multinational enterprises and a more global vision of the planet, we easily find chief executive officers and top managers (decision-makers) spread around the world, in different countries and with different time zones. Because of this, time and location pose as two major constraints to support group decision-making [5, 8].

To provide an answer and operate correctly in this type of scenarios, traditional GDSS have evolved to what we identify today as Web-based Group Decision Support Systems (Web-based GDSS). Web-based GDSS support the decision-making process by using main characteristics of ubiquity ("anytime" and "anywhere") $[9,10]$. This evolution follows the evolution of general technology, as well as to the need of enhancing the efficiency of the group decision-making processes. There are some works in the literature that address the term of Web-based GDSS [9]. The Web-based GDSS may present different complexity levels. They can provide information about decision-maker preferences and other simple statistical information [11, 12]. The current challenge is to develop systems that can properly support the group decisionmaking process when decision-makers are dispersed [13-16]. For this, it is essential that each decision-maker can correctly define his preferences and intentions for each problem. After that, the system should support and guide that decision-maker throughout the entire decision-making while providing all the relevant information at the most adequate times. Otherwise, the decision-maker may never trust the information provided by system which is critical for its overall success. Therefore, it makes sense to think in multiple criteria decision analysis (MCDA) [17] which allows decision-makers to share information through problem configurations [18] and is appropriate to deal with both complex decision problems that involve the interaction of decision-makers with conflicting opinions and also deal with multiple qualitative and quantitative objectives $[19,20]$.

In this paper, we proceed with our ongoing research in the context of Web-based GDSS by examining one of the many aspects that can affect group decision-making which is the definition of negative preferences while modelling user preferences. We take advantage of the model proposed in [21] and instead of only suggesting the most adequate alternatives to each decision-maker based on each decision-maker criteria preferences' configurations we formulated a model that can suggest the rejection of alternatives whenever certain situations are verified. With this model it is possible to present more relevant information throughout the decision-making process and this way the system can guide decision-makers in the achievement of more consensual and satisfactory decisions.

The rest of the paper is organized as follows: Section 2 describes the proposed model as well as all the considered steps, and in Section 3 we present some conclusions and some guidelines regarding future work that we aim to carry on. 


\section{$2 \quad$ Method}

As mentioned before, the framework proposed in this work is a variant of the Cognitive Analytic Process method which was previously presented in [21] and has been formulated in order to consider the possibility of suggesting alternatives to be rejected (eliminated) by decision-makers throughout the decision-making process. The model is divided in six main steps: the first step is the definition of the multi-criteria problem; the second step is the definition of the weights associated to each alternative and criterion; the third step is the adjustment of the weights associated with the alternatives to consider credibility, expertise and styles of behavior (these notions have been previously introduced in $[22,23])$; the fourth step is the classification of each criterion and the classification of each alternative based on the new adjusted values; the fifth step is the selection of the alternatives that have the worst classification; the last step is the measurement of the consistency for each selected alternative.

\subsection{Step 1: Multi-criteria problem definition}

In the first step the multi-criteria problem is defined, which includes criterion, alternative and decision-maker definitions.

Definition 1: Let $D$ be a decision matrix, $D=A * C$ where:

- $C$ is a set of criteria $C=\left\{c_{1}, c_{2}, \ldots, c_{n}\right\}, n>0$;

- $A$ is a set of alternatives $A=\left\{a_{1}, a_{2}, \ldots, a_{m}\right\}, m>0$.

Rule 1: $\forall a_{i} \in A, \forall c_{j} \in C, c_{j_{a_{i}}} \in \mathrm{D}$, each alternative $a_{i} \in A$ is related with each criterion $c_{j} \in C$. There cannot be an existing alternative with values for criteria that are not considered in the problem.

Definition 2: A criterion $c_{i}=\left\{i d_{c_{i}}, v_{c_{i}}, m_{c_{i}}\right\}$ consists of:

- $\forall c_{i} \in C, i \in\{1,2, \ldots, n\}$;

- $i d_{c_{i}}$ is the identification of a particular criterion;

- $v_{c_{i}}$ is the value of a particular criterion (Numeric, Boolean or Classificatory);

- $m_{c_{i}}$ is the greatness associated with the criterion (Maximization or Minimization).

Definition 3: An alternative $a_{i}=\left\{i d_{a_{i^{\prime}},}\left[c_{1_{a_{i}}}, c_{2_{a_{i}}}, \ldots, c_{n_{a_{i}}}\right]\right\}$ consists of:

- $\forall a_{i} \in A, i \in\{1,2, \ldots, m\}$;

- $i d_{a_{i}}$ is the identification of a particular alternative;

- $\left[c_{1_{a_{i}}}, c_{2_{a_{i}}}, \ldots, c_{n_{a_{i}}}\right]$ is the instantiation of each criterion.

Definition 4: Let $D^{\prime}$ be a normalized decision matrix such that: $\forall a_{i} \in A_{D^{\prime}} \wedge$ $\sum_{n=1}^{\left\langle C_{D^{\prime}}\right\rangle} c_{n_{a_{i}}}=1$. 
Definition 5: Let $D M$ be a set of decision-makers where $D M=\left\{d m_{1}, d m_{2}, \ldots, d m_{k}\right\}$ and $k \in\{1,2, \ldots, n\}$.

\subsection{Step 2: Definition of alternatives and criteria weights}

For the second step it will be defined the weight given by each decision-maker towards each alternative and each criterion. This will result in two preference matrices containing all the weights for each alternative and criterion.

Definition 6: Let $w_{d m_{i_{a_{j}}}}$ be the weight or preference given to a certain alternative $a_{j}$ by a decision-maker $\mathrm{dm}_{\mathrm{i}}$ and $a_{j} \in A$.

Rule 2: A decision-maker $d m_{i}$ can define a set of alternatives weights where:

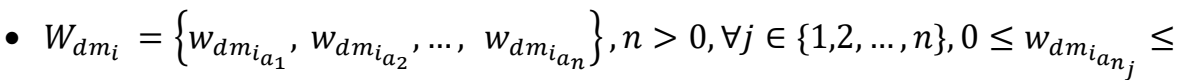
1

- $\left\langle W_{d m_{i}}\right\rangle=\langle A\rangle$.

Definition 6.1: Let $w c_{d m_{i} c_{j}}$ be the weight or preference given to a certain criterion $c_{j}$ by a decision-maker $d m_{i}$ and $c_{j} \in C$.

Rule 2.1: A decision-maker $\mathrm{dm}_{\mathrm{i}}$ can define a set of criteria weights where:

- $W C_{d m_{i}}=\left\{w c_{d m_{i_{c_{1}}}}, w c_{d m_{i_{c_{2}}}}, \ldots, w c_{d m_{i_{c_{n}}}}\right\}, n>0, \forall j \in\{1,2, \ldots, n\}, 0 \leq$ $w c_{d m_{i_{c_{j}}}} \leq 1$

- $\left\langle W C_{d m_{i}}\right\rangle=\langle C\rangle$.

Definition 7: Let $W_{D M}$ be the set of alternatives weights of a set of decision-makers $D M$ where: $W_{D M}=\left\{W_{d m_{1}}, W_{d m_{2}}, \ldots, W_{d m_{z}}\right\}, z>1$.

Definition 7.1: Let $W C_{D M}$ be the set of alternatives weights of a set of decision-makers $D M$ where: $W C_{D M}=\left\{W C_{d m_{1}}, W C_{d m_{2}}, \ldots, W C_{d m_{z}}\right\}, z>1$.

Definition 8: Let $A P_{D M}$ be an alternatives preference matrix, where:

$$
A P_{D M}=A \times W_{D M}=\left[\begin{array}{cccc}
w_{d m_{1 a_{1}}} & w_{d m_{2 a_{1}}} & \ldots & w_{d m_{i_{a_{1}}}} \\
w_{d m_{1 a_{2}}} & w_{d m_{2 a_{2}}} & \ldots & w_{d m_{i a_{2}}} \\
\vdots & \vdots & \ldots & \vdots \\
w_{d m_{1 a_{m}}} & w_{d m_{2 a_{m}}} & \ldots & w_{d m_{i_{a_{m}}}}
\end{array}\right]
$$

Definition 8.1: Let $C P_{D M}$ be a criteria preference matrix, where: 


$$
C P_{D M}=C \times W C_{D M}=\left[\begin{array}{cccc}
w c_{d m_{1}} & w c_{d m_{2} c_{1}} & \ldots & w c_{d m_{i_{c_{1}}}} \\
w c_{d m_{1}} & w c_{d m_{2}} & \ldots & w c_{d m_{c_{2}}} \\
\vdots & \vdots & \ldots & \vdots \\
w c_{d m_{c_{2}}} & w c_{d m_{2} c_{m}} & \ldots & w c_{d m_{i_{c_{m}}}}
\end{array}\right]
$$

\subsection{Step 3: Adjustment of alternatives weights using credibility and expertise of decision-makers}

In this step, the weight given for each alternative is readjusted with the credibility, style of behavior and expertise values of each decision-maker [22, 23]. The weight given for each alternative is used as well as the $A P_{D M c r e d i b l e}$ matrix that contains all the weights given for each alternative by the decision-makers that are credible.

Formula 1 correlates the style of behavior with the credibility using the values of concern for self and concern for others that have been selected by the decision-maker. Formula 2 readjusts the value obtained in Formula 1 according to the expertise level of the decision-maker. The expertise levels considered where defined in [23] and are Expert, High, Medium, Low and Null.

Definition 9: Let DMcredible ${ }_{d m_{i}}$ be the set of decision-makers that decision-maker $d m_{i}$ considers as credible.

We can now define Formula 1 which correlates the style of behavior with the credibility values:

$$
\forall d m_{i} \in D M, \forall a_{j} \in A, w_{d m_{i_{a_{j}}}}=\frac{w_{d m_{i_{a_{j}}}} \times C S_{d m_{i}}+\left(\frac{T P}{N D}\right) \times C O_{d m_{i}}}{C S_{d m_{i}}+C O_{d m_{i}}}(1)
$$

Where:

- $w_{d m_{i}}$ is the weight given to the alternative $a_{j}$ by decision-maker $d m_{i}$;

- $C S_{d m_{i}}$ is the value of the Concern for Self of decision-maker $d m_{i}$ chosen style of behavior;

- TP is the sum of the given weights to alternative $a_{j}$ by each one of the credible decision-maker in DMcredible $_{d m_{i}}$;

- $N D$ is the number of credible decision-makers such that $N D=\left\langle\right.$ DMcredible $\left._{d m_{i}}\right\rangle$;

- $C O_{d m_{i}}$ is the value of the Concern for Others of decision-maker $d m_{i}$ chosen style of behavior.

The weight of each alternative can now be readjusted with the decision-maker expertise level using Formula 2.

$$
\forall d m_{i} \in D M, \forall a_{j} \in A, w_{d m_{i_{a_{j}}}}=\frac{w_{d m_{i_{a_{j}}}} \times e_{d m_{i}}+\left(\frac{T P}{N D}\right) \times e_{d m_{i}}^{\prime}}{e_{d m_{i}}+e_{d m_{i}}^{\prime}}(2)
$$


Where:

- $w_{d m_{i_{a}}}$ is the readjusted weight given to alternative $a_{j}$ by decision-maker $\mathrm{dm}_{\mathrm{i}}$ using formula 1 ;

- $e_{d m_{i}}$ is the expertise level of decision-maker $d m_{i}$;

- $T P$ is the sum of the given weights to alternative $a_{j}$ by each one of the credible decision-makers in DMcredible ${ }_{d m_{i}}$;

- $N D$ is the number of credible decision-makers such that $N D=\left\langle\right.$ DMcredible $\left._{d m_{i}}\right\rangle$;

- $e^{\prime}{ }_{d m_{i}}$ is the inverse of expertise level of decision-maker $d m_{i}$.

\subsection{Step 4: Alternatives classification}

After the weight of each alternative has been readjusted using both Formula 1 and Formula 2 we can now classify both alternatives and criterions. For this we first define a $F_{D i f}$ function which return the difference between the maximum and minimum weights found in a $W$ weight set.

Definition 10: Let $F_{D i f}$ be a function that returns the difference between maximum and minimum weights given to the alternatives or criteria that belong to a set of $\mathrm{W}$ weights.

$$
F_{D i f}: W=\left\{\begin{array}{c}
\max (W)-\min (W), \text { if } \max (W) \neq \min (W) \\
\max (W)
\end{array}\right.
$$

This means that $F_{D i f}\left(W_{D M}\right)$ returns the difference between the alternative with the greatest weight and the alternative with the lowest weight, while for $F_{D i f}\left(W C_{D M}\right)$ returns the difference between the criterion with the greatest weight and the criterion with the lowest weight. The result of $F_{D i f}$ can now be classified in five different levels according Table 1.

Table 1. $F_{D i f}$ levels

\begin{tabular}{lc}
\hline Level $(\boldsymbol{l})$ & $\boldsymbol{F}_{\boldsymbol{D i f}}$ \\
\hline 5 & $\geq 0,80$ \\
4 & $\geq 0,60$ \\
3 & $\geq 0,40$ \\
2 & $\geq 0,20$ \\
1 & $<0,20$ \\
\hline
\end{tabular}

Since each criteria or alternative weighting is done in a scale of $[0,1]$ the minimum difference between two criteria or alternatives is less than 0.2 and the maximum difference is greater than 0.8. Measuring the difference (using function $F_{D i f}$ ) between the criterion or alternative with more weight and the criterion or alternative with less weight we can obtain (according to Table 1 ) the $l$ value.

After identifying the $l$ value we can then perform Algorithm 1 to measure the classification done for each criterion $i m p_{c_{j_{d m_{i}}}}$ or alternative $i m p_{a_{j_{d m_{i}}}}$. 


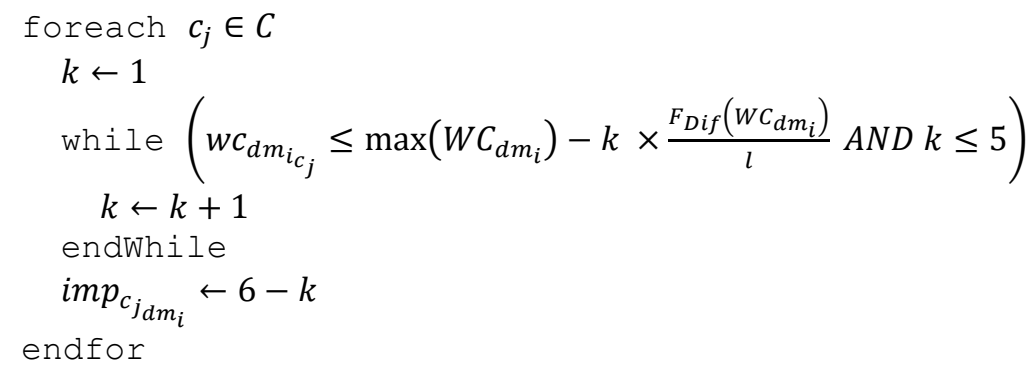

Algorithm 1. Criterion importance classification algorithm

To classify the importance of each alternative Algorithm 1 is also performed with the only difference being that the algorithm is applied for each $a_{j} \in A$ and the weights considered belong to the set $W_{D M}$. After $i m p_{c_{j_{d m_{i}}}}$ and $i m p_{a_{j_{d m_{i}}}}$ have been identified for each criterion and alternative we can now classify them according the assigned value as can be seen in Table 2 .

Table 2. Importance Classification

\begin{tabular}{lll}
\hline Value & $\boldsymbol{i m p}$ & Definition \\
\hline 5 & VI & Very Important \\
4 & I & Important \\
3 & M & Medium \\
2 & NI & Not Important \\
1 & IN & Insignificant \\
\hline
\end{tabular}

\subsection{Step 5: Alternatives Selection}

In the fifth step it will be identified the alternatives with the worst classification among all the classifications for each decision-maker. For this a set of $A I M P_{d m_{i}}$ must first be defined containing all the importance values for each alternative for the decision-maker $d m_{i}$.

Definition 11: Let $A I M P_{d m_{i}}$ be the set of importance values of all the alternatives for the decision maker $d m_{i}$, where:

- $A I M P_{d m_{i}}=\left\{i m p_{a_{1 m_{i}}}, i m p_{a_{2} d m_{i}}, \ldots, i m p_{a_{j_{d m_{i}}}}\right\}, j>0$;

- $\left\langle A I M P_{d m_{i}}\right\rangle=\langle A\rangle$.

Definition 11.1: Let $C I M P_{d m_{i}}$ be the set of importance values of all the criteria for the decision maker $d m_{i}$, where:

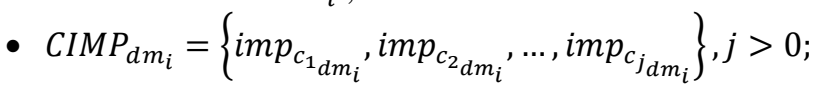

- $\left\langle C I M P_{d m_{i}}\right\rangle=\langle C\rangle$. 
We can now define the matrix $A E_{D M}$ which contains all the alternatives importance for all decision-makers.

Definition 12: Let $A E_{D M}$ be an alternatives evaluation matrix, where:

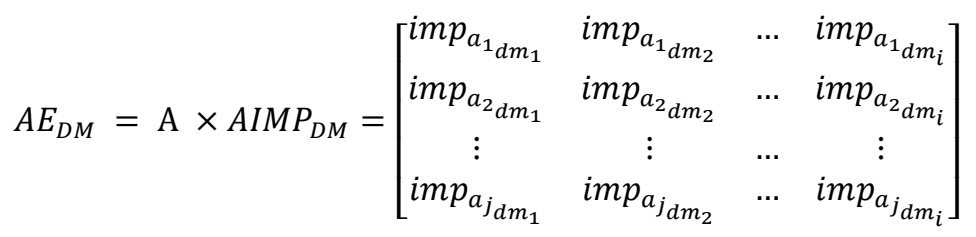

value $\leftarrow 1$

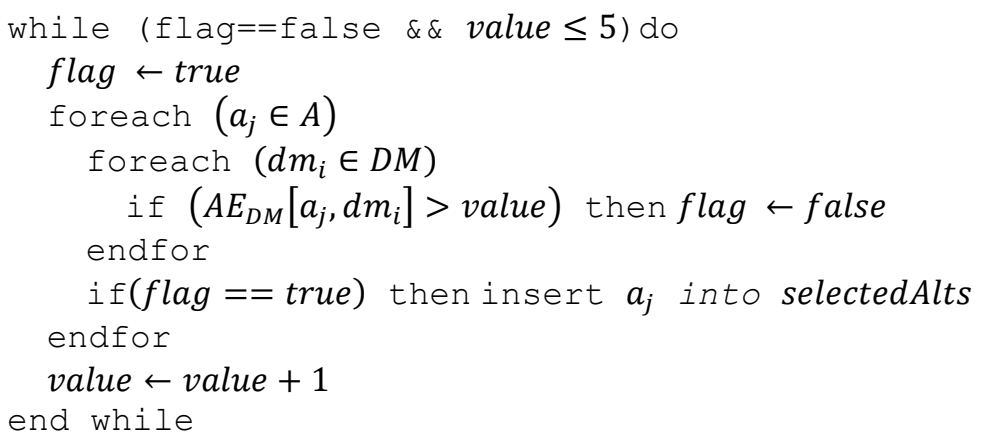

Algorithm 2. Alternative selection algorithm

After the evaluation matrix $A E_{D M}$ has been defined we can now perform Algorithm 2 which selects the alternatives with the worst classification selectedAlts based on its importance value. Algorithm 2 iterates through the entire $A E_{D M}$ matrix and first searches through all the alternatives importance values and attempts to find at least an alternative whose importance has the lowest possible value (which corresponds to Insignificant classification) for all the decision-makers. If an alternative is not found, the algorithm reiterates again with an increased value and the process is repeated until at least an alternative has been found. This means that in the worst case scenario all the alternatives would have been classified as Very Important by at least one of decisionmakers. In this case all the alternatives will be selected for Step 6.

\subsection{Step 6: Alternatives weighting consistency}

In the last step of the proposed model the worst alternative will be identified among the alternatives that were selected in the previous step (selectedAlts).

To identify the worst alternative, it is necessary to measure the consistency between the weight given to the alternative by each decision-maker and their preference for each criterion. For this we use the normalized values from the $D^{\prime}$ matrix and we classify the instantiation of each criterion for each alternative using the same process applied to Step 4. As a result, we will have a set $C I M P_{a_{j}}$ which contains the importance of each criterion for the alternative $a_{i}$ and is defined as follows: 
Definition 13: Let $\mathrm{CIMP}_{a_{j}}$ be the set of importance values of all the criteria for the alternative $a_{i}$, where:

- $\mathrm{CIMP} P_{a_{j}}=\left\{i m p_{c_{1_{a_{j}}}}, i m p_{c_{1_{a_{j}}}}, \ldots, i m p_{c_{i_{a_{j}}}}\right\}, j>0$;

- $\left\langle\mathrm{CIMP} \mathrm{a}_{a_{j}}\right\rangle=\langle C\rangle$.

After this, we define a function $F_{\text {Consistency }}$ which returns the difference between the importance of a criterion to a decision-maker $d m_{i}$ and the importance value of the same criterion for an alternative $a_{j}$.

Definition 14: Let $F_{\text {Cons }}$ be a function that returns the difference between the importance of a criterion $c_{i}$ to a decision-maker $d m_{k}$ and the importance value of the same criterion for an alternative $a_{j}$.

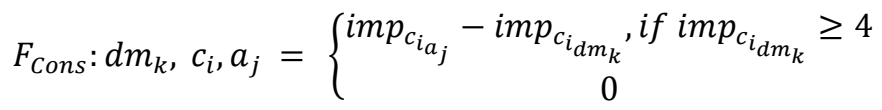

It is important to note that $F_{\text {Cons }}$ only returns the difference of criteria that are weighted as Very Important or Important by the decision-maker (value must be 4 or 5). This is done to make sure that the evaluation done to each alternative by the decisionmaker is consistent with the criteria that decision-maker considers to be important.

We can then define the consistency matrix $C M_{d m_{k}}$ of the decision-maker $d m_{k}$ as:

Definition 15: Let $C M_{d m_{k}}$ be a consistency matrix of the decision-maker $d m_{k}$, where:

$$
C M_{d m_{k}}=\left[\begin{array}{cccc}
F_{\text {Cons }}\left(d m_{k}, c_{1}, a_{1}\right) & F_{\text {Cons }}\left(d m_{k}, c_{2}, a_{1}\right) & \ldots & F_{\text {Cons }}\left(d m_{k}, c_{i}, a_{1}\right) \\
F_{\text {Cons }}\left(d m_{k}, c_{1}, a_{2}\right) & F_{\text {Cons }}\left(d m_{k}, c_{2}, a_{2}\right) & \ldots & F_{\text {Cons }}\left(d m_{k}, c_{i}, a_{2}\right) \\
\vdots & \vdots & \ldots & \vdots \\
F_{\text {Cons }}\left(d m_{k}, c_{1}, a_{j}\right) & F_{\text {Cons }}\left(d m_{k}, c_{2}, a_{j}\right) & \ldots & F_{\text {Cons }}\left(d m_{k}, c_{i}, a_{j}\right)
\end{array}\right]
$$

Finally, we define a function $F_{\text {TotalConsistency }}$ which returns the sum of all consistency values for an alternative $a_{j}$ of the decision-maker $d m_{k}$.

Definition 16: Let $F_{\text {TotalConsistency }}$ be a function which returns the sum of all consistency values for an alternative $a_{j}$ of the decision-maker $d m_{k}$.

$$
F_{\text {TotalConsistency }}: d m_{k}, a_{j}=\sum_{i=1}^{\langle C\rangle} F_{\text {Con }}\left(d m_{k}, c_{i}, a_{j}\right)
$$

The average consistency value $a c v$ between all $F_{\text {TotalConsistency }}$ for each decisionmaker is then measured according to Formula 3:

$$
\forall a_{j} \in A, a c v=\frac{\sum_{k=1}^{\langle D M\rangle} F_{\text {TotalConsistency }}\left(d m_{k}, a_{j}\right)}{D M}(3)
$$

Where: 
- $D M$ is the list of all decision-makers.

After the average consistency value is measured for each alternative in selectedAlts, the alternative with the lowest average consistency value is then suggested to all decision-makers as an alternative that should be rejected.

\section{Conclusions and Future Work}

As organizations and technology evolve, GDSS have also changed to be able to support decision-makers in a context where trying to establish a face-to-face meeting to deal with a problem has become a rather difficult task. However, the new Web-based GDSS have not been well accepted by organizations and we know from our previous works that the interaction between decision-makers and the system is an essential point regarding the acceptance of systems by users and the organizations themselves.

The model here proposed is a variant of a work that has been previously proposed and we define how we can measure the consistency between the assessment done by the decision-maker towards each alternative and the evaluation that was expected according to the assessment the decision-maker provided towards each criterion. This measure is essential to help us understand the impact that subjectivity has in the assessment done by the decision-maker. By identifying inconsistent alternatives assessment according to the criteria that a decision-maker considers to be important or very important, it will be possible to inform the decision-maker that the assessment provided was not the correct one and that there may be situations where it makes sense to reject a certain alternative. Likewise, there may be situations where the opposite situation is verified which means that a user might have provided a lower assessment than the expected one and his preference for a certain alternative should be increased.

Another important remark of this work is the inclusion of factors such as the credibility, expertise and styles of behavior which are part of the interactions that happen in real situations and that should be considered to better support the decisionmaking process.

Finally, but not less important, all the information that is measured using this model, such as the identification of very important alternatives or criteria or the identification of inconsistent and consistent evaluations can be easily provided to the decision-maker at any moment of the decision-making process. This will guide him and the group to obtain more consensual and satisfactory decisions. Furthermore, the system will also be ready to suggest the best decision at any moment of the decision-making process.

As future work, the first step is to evaluate the proposed model using a real case study in order to understand the impact that inconsistencies and that the rejection of alternative have in the achievement of more consensual and satisfactory decisions (compared to models that do not consider these concepts). Another point worth of studying is to consider the possibility of suggesting the reevaluation of preferences whose assessment should have been higher by decision-maker and its impact in the consensus and satisfaction that can be obtained. We also intend to study and include the concept of restrictions which the decision-maker can define throughout the decision-making process and that will let him accept or reject a certain alternative. 
Acknowledgments. This work was supported by NIS Project (ANI|P2020 21958) and has received funding from FEDER Funds through P2020 program and from National Funds through FCT - Fundação para a Ciência e a Tecnologia (Portuguese Foundation for Science and Technology) under the projects UID/EEA/00760/2013 and UID/CEC/00319/2013.

\section{References}

1. Lunenburg, F.C.: Decision making in organizations. International journal of management, business, and administration 15, 1-9 (2011)

2. Wilson, D.C.: Decision-making in organizations. Managing organizations: Current issues 43 (1999)

3. Luthans, F.: Organizational behavior. McGraw-Hill/Irwin 46, 594 (2011)

4. Dennis, A.R.: Information Exchange and Use in Group Decision Making: You Can Lead a Group to Information, but You Can't Make It Think. MIS Quarterly 20, 433-457 (1996)

5. Lunenburg, F.C.: Group decision making. In: National Forum of Teacher Education Journal, pp. 1-7. (2010)

6. Proctor, T.: Creative problem solving: Developing skills for decision making and innovation. New York, NY: Routledge (2011)

7. Gunnarsson, M.: Group decision making. Verlag, Frederick, MD (2010)

8. Huber, G.P.: Issues in the Design of Group Decision Support Systems. MIS Quarterly: Management Information Systems 8, 195-204 (1984)

9. Kwon, O., Yoo, K., Suh, E.: UbiDSS: a proactive intelligent decision support system as an expert system deploying ubiquitous computing technologies. Expert systems with applications 28, 149-161 (2005)

10.Morente-Molinera, J.A., Wikström, R., Herrera-Viedma, E., Carlsson, C.: A linguistic mobile decision support system based on fuzzy ontology to facilitate knowledge mobilization. Decision Support Systems 81, 66-75 (2016)

11.JAY F NUNAMAKER, J.: Future research in group support systems: needs, some questions and possible directions. International Journal of Human-Computer Studies 47, 357-385 (1997)

12.Shim, J.P., Warkentin, M., Courtney, J.F., Power, D.J., Sharda, R., Carlsson, C.: Past, present, and future of decision support technology. Decision support systems 33, 111-126 (2002)

13.Carneiro, J., Martinho, D., Marreiros, G., Novais, P.: Intelligent negotiation model for ubiquitous group decision scenarios. Frontiers of Information Technology \& Electronic Engineering 17, 296-308 (2016)

14.Sanchez-Anguix, V., Julian, V., Botti, V., García-Fornes, A.: Tasks for agent-based negotiation teams: Analysis, review, and challenges. Engineering Applications of Artificial Intelligence 26, 2480-2494 (2013)

15.Sánchez-Anguix, V., Botti, V., Julián, V., García-Fornes, A.: Analyzing intra-team strategies for agent-based negotiation teams. In: The 10th International Conference on Autonomous Agents and Multiagent Systems-Volume 3, pp. 929-936. International Foundation for Autonomous Agents and Multiagent Systems, (2011)

16.Carneiro, J., Martinho, D., Marreiros, G., Jimenez, A., Novais, P.: Dynamic argumentation in UbiGDSS. Knowledge and Information Systems 1-37 (2017) 
17.Greco, S., Figueira, J., Ehrgott, M.: Multiple criteria decision analysis. Springer's International series (2005)

18.Dehe, B., Bamford, D.: Development, test and comparison of two Multiple Criteria Decision Analysis (MCDA) models: A case of healthcare infrastructure location. Expert Systems with Applications 42, 6717-6727 (2015)

19.Ram, C., Montibeller, G., Morton, A.: Extending the use of scenario planning and MCDA for the evaluation of strategic options. Journal of the Operational Research Society 62, 817-829 (2011)

20.Golmohammadi, D., Mellat-Parast, M.: Developing a grey-based decision-making model for supplier selection. International Journal of Production Economics 137, 191-200 (2012)

21.Carneiro, J., Conceição, L., Martinho, D., Marreiros, G., Novais, P.: Including cognitive aspects in multiple criteria decision analysis. Annals of Operations Research 1-23 (2016)

22.Carneiro, J., Saraiva, P., Martinho, D., Marreiros, G., Novais, P.: Representing decisionmakers using styles of behavior: An approach designed for group decision support systems. Cognitive Systems Research (2017)

23.Carneiro, J., Martinho, D., Marreiros, G., Novais, P.: Including Credibility and Expertise in Group Decision-Making Process: An Approach Designed for UbiGDSS. In: World Conference on Information Systems and Technologies, pp. 416-425. Springer, (2017) 